\title{
RADICAL DECOMPOSITIONS OF IDEMPOTENT ALGEBRAS
}

\author{
B. J. GARDNER \\ (Received 24 September 1982; revised 11 February 1983) \\ Communicated by R. Lidl
}

\begin{abstract}
A variant of Kurosh-Amitsur radical theory is developed for algebras with a collection of (finitary) operations $\omega$, all of which are idempotent, that is satisfy the condition $\omega(x, x, \ldots, x)=x$. In such algebras, all classes of any congruence are subalgebras. In place of a largest normal radical subobject, a largest congruence with radical congruence classes is considered. In congruence-permutable varieties the parallels with conventional radical theory are most striking.
\end{abstract}

1980 Mathematics subject classification (Amer. Math. Soc.): 08 A 05, 08 C 99, 20 M 11.

\section{Introduction}

Since its introduction for rings in the early 50's, Kurosh-Amitsur radical theory has been developed for many types of algebraic and topological-algebraic structures-groups, topological rings and groups, modules, lattice-ordered rings, etc. - which form categories about as "nice" as categories of rings. (Though the module version of the theory had, to some extent, a separate incarnation as "torsion theory", it does belong in this list.)

A radical class of structures like the ones listed is a non-empty homomorphically closed class $R$ such that every object $A$ has a largest normal $\Re$-subobject $\Re(A)$, and $|\Re(A / \Re(A))|=1$ for every $A$.

The most obvious obstacle to an extension of the theory to other kinds of algebraic structure is the absence of a satisfactory notion of normal subobject. Some work has been done with semigroups with zero and with monoids. In the

C 1984 Australian Mathematical Society $0263-6115 / 84 \$ A 2.00+0.00$ 
former case, ideals play the part of normal subobjects: see Szász [28] for a "literal" generalization of some basic results; there is also a quite extensive literature (see for example [18]) dealing with another version, in which, rather than homomorphic closure, only closure under Rees factor semigroups is imposed on a radical class. In the monoid case, inverse images of the identity play the special role; see Márki et al. [19].

It seem clear, however, that wide-ranging generalizations necessitate the replacement of the subobject $R(A)$ by a suitable congruence on $A$. Some efforts have been made in this direction: see Hoehnke [12], Mlitz [20] and Strecker [27]. These are largely based on Hoehnke's notion of an $M$-radical [12]. What is sought is a congruence $\rho_{A}$ associated with a class $\Re$ for each object $A$ such that $A / \rho_{A}$ has no congruence for which some nontrivial class is an object of $\Re$ and, if possible, $\rho_{A}$ contains every congruence on $A$ having a class which is an object of $R$. Complications can arise if there are no congruence classes which are subobjects.

(We note in passing that another kind of radical, a subfunction $r$ of the identity such that $f(r(A)) \subseteq r(f(A))$ for homomorphisms $f$ and $|r(A) / r(A)|=1$ for all $A$ has an exact analogue in any setting; see, for example, Clifford [4] or Hoehnke [12].)

In this paper we consider only varieties of idempotent algebras: those for which every (finitary) operation $\omega$ satisfies the condition

$$
\omega(x, x, \ldots, x)=x .
$$

Equivalently, we are dealing with algebras in which every one-element subset is a subalgebra. Equivalently again, our algebras are those in which every class of every congruence is a subalgebra.

In defining radical classes $\Re$ in terms of associated congruences $\rho_{A}$, we have a choice: either (i) require every $\rho_{A}$-class to be in $\Re$ and $A / \rho_{A}$ to have no non-trivial congruence with all classes in $\Re$ or (ii) require that $A / \rho_{A}$ have no congruence with a non-trivial class in $R$, and correspondingly require that $\rho_{A}$ be larger than any congruence on $A$ with a non-trivial class in $R$. Both might be regarded as generalizations of the "classical" version. Motivated in large part by examples like the semilattice decomposition of a band, we have opted for (i). Radical classes can in fact satisfy both (i) and (ii); they may then be viewed as resembling strict or strong radical classes of rings.

Thus every algebra $A$ will have a congruence $\rho_{A}$, all of whose classes are "radical" algebras and such that $A / \rho_{A}$ is a "semi-simple" algebra, hence the term radical decomposition. Borrowing a convention from semigroup theory, we could say that every algebra is a semi-simple algebra of radical algebras.

In radical theory for rings (and the like) the following characterization is very useful. $A$ class $\Re$ is a radical class if and only if

(i) $\Re$ is homomorphically closed; 
(ii) $\Re$ is closed under extensions, that is, if $I \triangleleft R$ and if $I, R / I \in \mathscr{R}$, then $R \in \mathscr{R}$ and

(iii) $\Re$ contains all unions of chains of $\Re$-ideals.

To show that every ring has a largest (and not just maximal) $\Re$-ideal, one uses (ii) in conjunction with the isomorphism theorem $(I+J) / J \cong I / I \cap J$. In some situations where we wish to discuss radical theory, there is no analogue of this isomorphism and we need other conditions besides the analogues of (i), (ii) and (iii), to be sure of getting radical classes.

An important case where a form of this isomorphism is valid is where congruences permute, that is, $\sigma \circ \tau=\tau \circ \sigma$, where we define

$$
a(\sigma \circ \tau) b \Leftrightarrow \exists c \text { such that } a \sigma c \text { and } c \tau b \text {. }
$$

In fact, throughout the paper, we prove two kinds of result: the general version and the nicer, more complete version for congruence-permutable varieties.

Another point at which we have to make this distinction is in the requirement of homomorphic closure for radical classes: when congruences do not permute something more complicated than homomorphic closure (which suffices for congruence-permutable varieties) is needed. In the general case, homomorphic closure alone produces what we call weak radical classes (see Section 4). (For an account of congruence-permutablility, see, for example, [3] Chapter II, Section 12.)

Varieties which are radical or semi-simple classes have been investigated previously. For rings (associative and otherwise) the former are the varieties which are closed under extensions, the latter the ones with attainable identities [9]. The situation is more complicated with idempotent algebras (see Section 3). Our version of extension-closure for a class $\mathcal{X}$ is as follows: if $A$ has a congruence $\sigma$ such that all $\sigma$-classes and $A / \sigma$ are in $\mathscr{X}$, then $A \in X$. For attainability of the identities of $\mathcal{W}$, we require that for every $A, \wedge\{\sigma \mid A / \sigma \in \mathcal{V}\}$ have no congruence classes with non-trivial images in $\mathcal{T}$. These are the definitions used by Tamura [30] and Mal'cev [17] Chapter 32 who first considered these concepts.

The plan of the paper is as follows: Section 1 introduces radical classes; Section 2 semi-simple classes; Section 3 treats varieties; Section 4 discusses weak radical classes; Section 5 is devoted to a version of the lower radical construction in congruence-permutable varieties; all examples are contained in Section 6.

We should observe that while our discussion is purely algebraic, our theory has some affinities with the generalized connectedness theory of topological spaces (Arhangel'skii and Wiegandt [1]) and graphs (Fried and Wiegandt [8]).

For universal algebraic terminology we refer the reader to [3], for semigroup theory to [13] and for radical-theoretic background to [33].

Throughout the paper, we shall always have as our universal class some variety of idempotent algebras. By "variety" we mean "subvariety of the universal 
class". One piece of not-quite-standard notation: the trivial congruence (that is, equality) on an algebra $A$ is denoted by $0_{A}$, the largest congruence by $1_{A}$ (that is, $a 1_{A} b$ for all $a, b \in A$ ).

\section{Radical classes}

This section introduces radical classes and provides partial characterizations of them, based on known characterizations of radical classes of rings.

1.1 Definition. A radical class is a non-empty class $\Re$ satisfying the following conditions.

(R1) If $\sigma$ and $\tau$ are congruences on an algebra $A$ such that all $\sigma$-classes are in $\Re$, then there is a congruence $\lambda$ on $A / \tau$ such that $(\sigma \vee \tau) / \tau \leqslant \lambda$ and all $\lambda$-classes are in $R$.

(R2) For every algebra $A$ there is a congruence $\rho_{A}$ on $A$ such that all $\rho_{A}$-classes are in $R$ and $\rho_{A} \geqslant \mu$ for every congruence $\mu$ on $A$ with all its classes in $R$.

(R3) $\rho_{A / \rho}=0_{A / \rho}$ for every $A$.

When convenient, we shall drop the subscript and write $\rho$ instead of $\rho_{A}$.

The following is obvious.

1.2 Proposition. Let $\mathcal{R}$ be radical class, $\rho$ the corresponding congruence. Then $\rho_{A}=1_{A}$ if and only if $A \in \Re$.

Property (R1) ensures that the congruence associated with a radical class is functorial in an appropriate weak sense.

1.3 Proposition. Let $\Re$ be a radical class with congruence $\rho$. For any surjective homomorphism $f: A \rightarrow B$, we have $f\left(\rho_{A}\right) \subseteq \rho_{B}$.

Proof. Let $\kappa=\operatorname{Ker}(f)$. Then if $a_{1} \rho_{A} a_{2}$, we have $a_{1}\left(\rho_{A} \vee \kappa\right) a_{2}$ and thus $f\left(a_{1}\right)\left(\left(\rho_{A} \vee \kappa\right) / \kappa\right) f\left(a_{2}\right)$, so by (R1) and (R2), $f\left(a_{1}\right) \rho_{B} f\left(a_{2}\right)$.

Note that by 1.3 and (R3), our radicals are radicals in the sense of Hoehnke [12].

We consider next some closure properties which provide alternative characterizations of radical classes. The first of these is a version of closure under extensions, the others relate to joins of congruences. Let $\mathscr{K}$ be a class of algebras. These are the properties, which $\mathscr{K}$ may or may not satisfy. 
(E) If an algebra $A$ has a congruence $\sigma$ such that all $\sigma$-classes and $A / \sigma$ belong to $\mathscr{K}$, then $A$ is in $\mathscr{K}$.

(C) If $\left\{\sigma_{\alpha} \mid \alpha \in \Lambda\right\}$ is a chain of congruences on an algebra $A$ such that each $\sigma_{\alpha}$-class is in $\mathscr{K}$ for each $\alpha$, then each $\vee \sigma_{\alpha \in \Lambda}$-class is in $\mathcal{K}$.

(J) If $\sigma, \tau$ are congruences on an algebra $A$ such that all $\sigma$-classes and all $\tau$-classes are in $\mathscr{K}$, then all $\sigma \vee \tau$-classes are in $\mathscr{K}$.

1.4 Proposition. Let $\Re$ be a radical class. Then $\Re$ satisfies $(\mathrm{E})$.

Proof. Let $A$ have a congruence $\sigma$ such that $R$ contains each $a \sigma$ and $A / \sigma$. Let $\rho$ be the congruence associated with $\Re$. Then $\sigma \leqslant \rho$, so $\bar{A}=A / \rho$ is a homomorphic image of $A / \sigma$, whence by (R1), $\bar{A} \in \Re$, that is, $\rho_{A}^{-}=1_{A^{-}}$. But $\rho_{A}^{-}=0_{A}^{-}$, so $\left|A / \rho_{A}\right|=|\bar{A}|=1$, that is, $\rho_{A}=1_{A}$, that is, $A \in \Re$.

1.5 Proposition. Let $\Re$ be a radical class. Then $\Re$ satisfies $(C)$.

Proof. Let $\left\{\sigma_{\alpha} \mid \alpha \in \Lambda\right\}$ be a chain of congruences on an algebra $A$ such that each $\sigma_{\alpha}$-class is in $\Re$ for each $\alpha$, and let $\sigma=\bigvee_{\alpha \in \Lambda} \sigma_{\alpha}$. Let $K=a \sigma$ be any $\sigma$-class. Then for each $b \in K$, we have

$$
K=b \sigma=\bigcup_{\alpha \in \Lambda} b \sigma_{\alpha} .
$$

Let $\tau_{\alpha}$ denote the restriction of $\sigma_{\alpha}$ to the subalgebra $K$. Clearly $\tau_{\alpha}$ is a congruence on $K$ and $b \tau_{\alpha}=b \sigma_{\alpha}$ for each $b \in K$. Thus each $\tau_{\alpha}$-class is in $R$, so that $\tau_{\alpha} \leqslant \rho_{K}$ for each $\alpha$, and hence $V_{\alpha \in \Lambda} \tau_{\alpha} \leqslant \rho_{K}$. But $\bigvee_{\alpha \in \Lambda} \tau_{\alpha}$ is the restriction to $K$ of $\sigma$ and this is $1_{K}$. Thus $K$ is in $R$.

1.6 THEOREM. Let $R$ be a non-empty class satisfying (R1), (E), (C) and (J). Then $\Re$ is a radical class.

Proof. We need to verify (R2) and (R3). Let $\mathcal{C}(A)$ be the set of congruences on $A$, all of whose classes are in $R$, and let $\left\{\sigma_{\alpha} \mid \alpha \in \Lambda\right\}$ be a chain in $\mathcal{C}(A)$. Then $\sigma=\bigvee_{\alpha \in \Lambda} \sigma_{\alpha} \in \mathcal{C}(A)$ by $(C)$, so $\mathcal{C}(A)$ has a maximal element, $\mu$. For any maximal member $\nu$ of $\mathcal{C}(A)$, we have $\mu \vee \nu \in \mathcal{C}(A)$ and thus $\mu=\nu$. Since (by a similar argument to the one already used) every congruence in $\mathcal{C}(A)$ is contained in a maximal one, $\mathcal{C}(A)$ has a largest element $\mu_{A}$. This gives us (R2).

Let $\vec{A}=A / \mu_{A}$ and let $\lambda$ be a congruence on $\bar{A}$, all of whose classes are in $R$. Let $\hat{\lambda}$ be the congruence on $A$ defined by

$$
a \hat{\lambda} b \Leftrightarrow(a \mu) \lambda(b \mu)
$$


and let $T$ be a congruence class of $\hat{\lambda}$. Since $\mu \leqslant \hat{\mu}$, we have

$$
T=\bigcup\{t \mu \mid t \in T\}
$$

Let $\mu^{\prime}$ denote the restriction of $\mu$ to $T$. Then all $\mu^{\prime}$-classes are $\mu$-classes, and therefore belong to $R$.

By construction, $\hat{\lambda} / \mu=\lambda$, so $T / \mu^{\prime}$, as a $\lambda$-class, is in $\Re$. By (E) we have $T \in \mathscr{R}$. This means that $\hat{\lambda} \in \mathcal{C}(A)$ and thus $\hat{\lambda} \leqslant \mu$, that is, $(a \mu) \lambda(b \mu)$ implies $a \mu b$, that is, $\lambda=0_{A / \mu}$. We therefore have (with the obvious interpretation) $\mu_{A / \mu}=0_{A / \mu}$. Thus (R3) is also satisfied.

It is appropriate to say a word or two at this point about Properties (R1) and (J). The former, which ensures the "functoriality" of the congruence $\rho$ associated with a radical class $\Re(1.3)$ is a strong form of homomorphic closure: if $A \in R$, then $\rho_{A}=1_{A}$, so for any congruence $\tau$ on $A, 1_{A / \tau}=1_{A} / \tau=\left(1_{A} \vee \tau\right) / \tau$ must be contained in a congruence with all its classes in $\Re$, that is, $A / \tau$ must be in $\mathscr{R}$. Property $(J)$, on the other hand, ensures, in the presence of $(C)$, that every algebra has a largest, rather than merely maximal congruence with its classes in $\mathscr{R}$. We do not in fact know of a class satisfying (Rl) (or homomorphic closure), (E) and (C) but not (J). For (R2) and (R3) plus homomorphic closure but without (R1), see Section 6.B. As we shall shortly see, in the presence of permutable congruences, we can dispense with $(J)$ and replace $(R 1)$ with homomorphic closure.

The following result is effectively given by Birkhoff ([2], pages $87-88$ ).

1.7 ThEOREM. Let $\sigma, \tau$ be permutable congruences on an algebra $A$. Then for any $a \in A$, we have

$$
a \sigma /(\sigma \wedge \tau) \cap(a \sigma \times a \sigma) \cong a(\sigma \vee \tau) / \tau \cap(a(\sigma \vee \tau) \times a(\sigma \vee \tau))
$$

1.8 COROLlaRY. In a variety with permutable congruences, a class $\mathscr{X}$ satisfies (R1), if and only if it is homomorphically closed.

Proof. Let $\mathscr{X}$ be homomorphically closed and let $\sigma, \tau$ be congruences on an

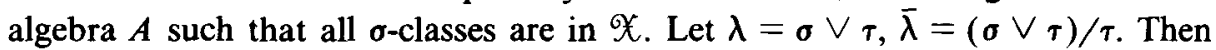
for $a \in A$, we have

$$
(a \tau) \bar{\lambda}=\{b \tau \mid a \lambda b\}=\{b \tau \mid b \in a(\sigma \vee \tau)\}
$$

Thus $(a \tau) \bar{\lambda}$ is the image of $a(\sigma \vee \tau)$ under the natural map from $A$ to $A / \tau$, that is, $(a \tau) \bar{\lambda} \cong a(\sigma \vee \tau) / \tau \cap(a(\sigma \vee \tau) \times a(\alpha \vee \tau))$. By 1.7, the latter is isomorphic to $a \sigma /(\sigma \vee \tau)$. Since $a \sigma \in \mathcal{X}$ and $\mathcal{X}$ is homomorphically closed, all congruence classes of $(\sigma \vee \tau) / \tau(=\bar{\lambda})$ are themselves in $\mathcal{X}$. Thus $\mathcal{X}$ satisfies (RI). The converse has already been noted. 
1.9 CoROLlaRY. If a class $R$ satisfies $(\mathrm{R} 1),(\mathrm{E}),(\mathrm{C})$ and

$\left(\mathrm{J}^{\prime}\right)$ If $\sigma, \tau$ are congruences on $A$ such that all $\sigma$-classes and all $\tau$-classes are in $R$, then $\sigma \circ \tau=\tau \circ \sigma$,

then $R$ is a radical class.

Proof. It is enough to show that $\left(\mathrm{J}^{\prime}\right)$ implies $(\mathrm{J})$. From the proof of 1.7 it can be seen that $\tau$ induces a congruence $\tau^{*}$ on $a(\sigma \vee \tau)$ such that all $\tau^{*}$-classes are $\tau$-classes and therefore belong to $\Re$. On the other hand, 1.7 also says that

$$
a(\sigma \vee \tau) / \tau^{*} \cong a \sigma /(\sigma \wedge \tau) \cap(a \sigma \times a \sigma),
$$

so $a(\sigma \vee \tau) / \tau^{*} \in \Re$ and hence, by $(\mathrm{E}), a(\sigma \vee \tau) \in \Re$.

For varieties with permutable congruences, we can prove an improved version of 1.6.

1.10 THEOREM. In a congruence-permutable variety, a non-empty class is a radical class if and only if it is homomorphically closed and satisfies (E) and (C).

Proof. If $\Re$ is a radical class, it satisfies the three stated conditions by $1.1,1.4$ and 1.5. Conversely, if the three stated conditions are satisfied, $R$ satisfies $(R 1)$ by 1.8. Thus by $1.9, \Re$ is a radical class.

It should be noted that the characterization of radical classes given by 1.10 is very closely analogous to a well known characterization of radical classes of rings, groups etc.

We conclude this section with a couple of characterizations of radical classes in special cases.

1.11 Definition. A class $\mathcal{X}$ of algebras is hereditary if for $A \in \mathscr{X}$ we have $a \sigma=\mathfrak{X}$ for every $a \in A$ and every congruence $\sigma$ on $A$.

1.12 PROPOSITION. A non-empty hereditary class $\mathcal{R}$ is a radical class if and only if it satisfies (R1), (E), (C) and (J).

Proof. By 1.6, 1.1, 1.4 and 1.5, we only have to show that hereditary radical classes satisfy (J). Let $\rho$ be the congruence associated with a hereditary radical class $\Re$. If $\sigma, \tau$ are congruences on an algebra $A$ such that all $\sigma$-classes and all $\tau$-classes are in $\Re$, then $\sigma \leqslant \rho, \tau \leqslant \rho$ and thus $\sigma \vee \tau \leqslant \rho$. Thus $\sigma \vee \tau$ induces a congruence on any $\rho$-class $T$, and all classes of this congruence are $\sigma \vee \tau$-classes. If $a \in T$, then since $T \in \Re$ and $\Re$ is hereditary, $a(\sigma \vee \tau) \in \Re$. But every $\sigma \vee \tau$-class is accounted for in this way. 


\section{Semi-simple classes}

2.1 Definition. A semi-simple class is a class $\mathcal{S}$ such that there exists a radical class $R$, with associated congruence $\rho$, for which

$$
\delta=\left\{A \mid \rho_{A}=0_{A}\right\} \text {. }
$$

The characterization of semi-simple classes in universal classes of rings has proved to be much more difficult than that of radical classes, though in some cases, for example associative and alternative rings, very satisfactory characterizations have been obtained.

We now obtain a characterization of semi-simple classes in an arbitrary variety of idempotent algebras. The description can be tidied up in the special case where congruences permute.

With any class $\mathscr{X}$ containing all one-element algebras, and every algebra $A$, we associate the congruence

$$
\kappa_{A}^{\mathfrak{X}}=\wedge\{\sigma \mid A / \sigma \in \mathfrak{X}\}
$$

2.2 THEOREM. $A$ non-empty class $\mathfrak{S}$ is a semi-simple class if and only if it satisfies the following:

(S0) All one element algebras are in $\mathfrak{S}$.

(S1) If $\tau$ is a congruence on an algebra $A$ and $A / \tau \in S$, then for any congruence $\sigma$ on $A$ such that $\sigma \$ \tau$, some $\sigma$-class has a non-trivial homomorphic image in $\mathcal{S}$.

(S2) $§$ is closed under subdirect products.

(S3) For every algebra $A$, we have $\kappa_{T}^{\S}=1_{T}$ for every $\kappa_{A}^{\S}$-class $T$.

Proof. Let $\Re$ be a radical class with associated congruence $\rho, \delta=\left\{A \mid \rho_{A}=0_{A}\right\}$. Since $\rho$ is a radical in the sense of Hoehnke [12] $\delta$ is subdirectly closed, that is, satisfies (S2). For any algebra $A$, we have $A / \rho_{A} \in \mathcal{S}$, so $\kappa_{A}^{\delta} \leqslant \rho$. By (S2) $A / \kappa_{A}^{\mathcal{\delta}} \in \mathfrak{S}$, so by (R1) the natural map $A \rightarrow A / \kappa_{A}^{\mathcal{\delta}}$ takes $\rho_{A}$-equivalent elements to $\mu$-equivalent elements, where $\mu$ is some congruence on $A / \kappa_{A}^{\delta}$ with all $\mu$-classes in $\Re$. This means that $\mu=0$ and so $\rho_{A} \leqslant \kappa_{A}^{\mathcal{S}}$. Thus we have $\rho_{A}=\kappa_{A}^{\mathfrak{\delta}}$.

Now let $\tau$ be a congruence on an algebra $A$ such that $A / \tau \in \mathcal{S}$. If $\sigma$ is a congruence on $A$ such that no $\sigma$-class $T$ has a non-trivial homomorphic image in $\S$, then for all such $T, \rho_{T}=\kappa_{T}=1_{T}$, so $T \in \mathscr{R}$. thus $\sigma \leqslant \rho_{A}=\kappa_{A}^{\S} \leqslant \tau$ and $\delta$ satisfies (S1).

If $T$ is a $\kappa_{A}$-class for some algebra $A$, then as noted above, $T$ is a $\rho_{A}$-class, so $\kappa_{T}=\rho_{T}=1_{T}$. Hence $\delta$ satisfies (S3).

We now turn to the converse. Let $\delta$ be non-empty and satisfy (S1), (S2) and (S3), and let

$$
\Re=\left\{A \mid A / \boldsymbol{\sigma} \in \mathcal{S} \Rightarrow \boldsymbol{\sigma}=1_{A}\right\} \text {. }
$$


Then $\Re=\left\{A \mid \kappa_{A}^{\S}=1_{A}\right\}$. By (S3), each $\kappa_{A}^{\varsigma}$-class is in $R$, for every algebra $A$; also $\bar{A}=A / \kappa_{A}^{\delta} \in \mathcal{S}$ by $(\mathrm{S} 2)$, so $\kappa_{A}^{-}=0_{\bar{A}}$.

Let $\sigma$ be a congruence on an algebra $A$ with all $\sigma$-classes in $R$, that is, such that no $\sigma$-class $T$ has a non-trivial homomorphic image in $\mathcal{S}$. By $(\mathrm{Sl})$,

$$
\sigma \leqslant \kappa_{A}^{\S}=\wedge\{\tau \mid A / \tau \in \delta\}
$$

By (S3) and (S2) $\kappa_{A}$ satisfies (R2) and (R3).

It is easy to see that $f\left(\kappa_{A}^{\S}\right) \subseteq \kappa_{B}^{\delta}$ for any surjective homomorphism $f: A \rightarrow B$. Thus $R$ satisfies (R1), so $R$ is a radical class, with $\kappa^{\S}$ as its associated congruence. The corresponding semi-simple class is $\left\{A \mid \kappa_{A}^{\varsigma}=0_{A}\right\}$, that is, $\delta$.

If a class $\mathcal{S}$ satisfies ( $\mathrm{S} 1$ ), then for $A \in \mathcal{S}$ we have $A / 0_{A} \in \mathcal{S}$, so every non-trivial congruence $A$ has a class with a non-trivial homomorphic image in $\delta$. This latter is a more reasonable (and familiar) looking condition. It is difficult to see, however, how the more cumbersome (S1) can be avoided in general. As usual, there is some simplification when congruences permute.

2.3 THEOREM. In a congruence-permutable variety, a non-empty class is a semi-simple class if and only if it satisfies (S2), (S3) and

$\left(\mathrm{S}^{\prime}\right)$ If $A \in \mathcal{S}$, then for every congruence $\sigma \neq 0_{A}$ on $A$, some $\sigma$-class has $a$ non-trivial homomorphic image in $\delta$.

Proof. We only have to show that (S1') implies (S1). Let $A / \tau$ be in $\delta$, and let $\sigma$ be a congruence on $A$ such that $\sigma \neq \tau$. Then $\tau<\sigma \vee \tau$ and $\bar{\sigma}=(\sigma \vee \tau) / \tau \neq 0_{A / \tau}$. Thus by $\left(\mathrm{Sl}^{\prime}\right)$, some $\bar{\sigma}$-class has a non-trivial homomorphic image in $\mathcal{S}$. But by the argument used in the proof of 1.8, each $(\sigma \vee \tau) / \tau$-class is a homomorphic image of a $\sigma$-class. Thus at least one $\sigma$-class can be mapped non-trivially onto something in $\S$; thus $\mathfrak{S}$ satisfies $(\mathbf{S} 1)$.

2.3 is as much as can be hoped for. In particular, it seems unlikely that (S3) can be dropped; its analogue is needed for non-associative rings, for example (see [21]).

\section{Varieties}

In the case of universal classes which are varieties of rings and related structures, a fair amount is now known about the (sub) varieties which are radical or semi-simple classes (see, for example, [9]). We next consider these questions for idempotent algebras. 
3.1 Theorem. $A$ (sub) variety $\mathcal{V}$ is a radical class if and only if it satisfies (R1), (E) and (J).

Proof. By 1.12 we need only observe that $\mathcal{V}$ satisfies (C). Let $\left\{\sigma_{\alpha} \mid \alpha \in \Lambda\right\}$ be a chain of congruences on an algebra $A$ such that all $\sigma_{\alpha}$-classes are in $\mathcal{V}$ for each $\alpha$. Let $\sigma=V_{\alpha \in \Lambda} \sigma_{\alpha}$. Since, for each $a \in A, a \sigma=\cup_{\alpha \in \Lambda} a \sigma_{\alpha}$ and varieties are closed under directed unions, we have $a \sigma \in \mathcal{T}$.

A variety $\mathcal{V}$ has attainable identities (Tamura [30]) if (in the sense of Section 2) Tsatisfies (S3).

3.2 TheOREM. $A$ (sub) variety $\mathcal{V}$ is a semi-simple class if and only if it has attainable identities.

Proof. Suppose $\mathcal{W}$ has attainable identities. Then we only need to prove that $\mathcal{T}$ satisfies (S1). To this end, let $\sigma$ be a congruence on $A$ such that no $\sigma$-class has a non-trivial homomorphic image in $\mathscr{V}$. Then the natural map $A \rightarrow A / \kappa_{A}^{\mathcal{V}}$ takes each $\sigma$-class $S$ to a subalgebra $\bar{S}$ of $A / \kappa_{A}^{\mathcal{V}}$. But as $\mathfrak{V}$ is a variety, we have $\bar{S} \in \mathcal{V}$. By attainability, $\kappa_{S}^{\mathcal{V}}=1_{S}$, whence $|\bar{S}|=1$. It follows that $\sigma \leqslant \kappa_{A}^{\tau} \leqslant \tau$ for all $\tau$ with $A / \tau \in \mathcal{V}$; thus (S1) is satisfied.

Conversely, if $\mathcal{T}$ is a semi-simple class, it has attainable identities by (S3).

A semi-simple radical class (SSR class), is a class which is at the same time a radical class and the semi-simple class corresponding to some other radical class. For varieties of rings and the like, a semi-simple class is an SSR class if and only if it's homomorphically closed, [10], [33]. Here we can prove the following.

3.3 TheOREM. A semi-simple class is an SSR class if and only if it satisfies (R1) and $(\mathrm{J})$.

Proof. Let $\delta$ be a semi-simple class satisfying (R1) and (J). By (R1) $\mathcal{S}$ is homomorphically closed, and by $2.2 \mathcal{S}$ is closed under subdirect products, so by Kogalovskii's Theorem [14], [22] $\mathcal{S}$ is a variety. By $3.2 \delta$ has attainable identities and so satisfies (E) ([17], page 443). It now follows from 3.1 that $\mathcal{V}$ is an SSR class.

Conversely, if $\mathcal{V}$ is an SSR class, then again $\mathcal{V}$ is homomorphically closed and closed under subdirect products, hence a variety as well as a radical class. By 3.1 V satisfies (J) (as well as (Rl)).

3.4 COROLlaRY. Every SSR class is a subvariety. 
In the presence of permutable congruences, we can state a neater result, in view of 1.10 and 3.1.

3.5 THEOREM. In a congruence-permutable variety, a semi-simple class is an SSR class if and only if it is homomorphically closed.

3.6 COROLLARY. In a congruence-permutable variety, a class is an SSR class if and only if it is a subvariety with attainable identities.

\section{Weak radical classes}

Condition ( $\mathrm{R} 1)$ in the definition (1.1) of a radical class is a rather cumbersome one compared with homomorphic closure which suffices in the case of congruence-permutable varieties. In this brief section we consider what happens in general when the weaker property is substituted.

4.1 Definition. A weak radical class is a non-empty homomorphically closed class satisfying (R2) and (R3) of 1.1 .

We shall see in Section 6.B below that there are weak radical classes which are not radical classes. The following result provides a further connection between these two concepts.

4.2 Proposition. Let $\Re$ be a weak radical class with associated congruence $\rho$. The following are equivalent:

(i) $R$ is a radical class.

(ii) $f\left(\rho_{A}\right) \subseteq \rho_{B}$ for every surjective homomorphism $f: A \rightarrow B$.

(iii) The class $\left\{A \mid \rho_{A}=0_{A}\right\}$ is closed under subdirect products.

Proof. (i) $\Leftrightarrow$ (ii): This follows by the arguments used on pages 6-7 of Clifford [4].

(i) $\Rightarrow$ (iii): 2.2 .

(iii) $\Rightarrow$ (ii): Let $\mathcal{S}=\left\{T \mid \rho_{T}=0_{T}\right\}$. For each $A$, let $\kappa_{A}^{\S}=\wedge\{\sigma \mid A / \sigma \in \delta\}$. Then $A / \kappa_{A}^{\mathcal{S}} \in \mathcal{S}$. Also $A / \rho_{A} \in \mathcal{S}$, so $\kappa_{A}^{\mathfrak{S}} \leqslant \rho_{A}$. But $\rho_{A} / \kappa_{A}^{\mathfrak{S}}$ is a congruence on $A / \kappa_{A}^{\mathfrak{S}}$ with its classes in $R$, so $\rho_{A}=\kappa_{A}^{\S}$. Let $f: A \rightarrow B$ be a surjective homomorphism. Then

$$
f\left(\rho_{A}\right)=f\left(\kappa_{A}^{\S}\right) \subseteq \kappa_{B}^{\mathfrak{S}}=\rho_{B} .
$$

4.3 TheOREM. A class o is a weak radical class if it is homomorphically closed and satisfies $(\mathrm{E}),(\mathrm{C})$ and $(\mathrm{J})$ of 1.6 . 
(See the proof of 1.6.)

4.4 THEOREM. In a congruence-permutable variety, all weak radical classes are radical classes.

4.5 THEOREM. $A$ (sub) variety is a weak radical class if and only if it satisfies (E) and $(\mathrm{J})$.

Proof. 3.1.

\section{The lower radical construction}

In this section we shall work entirely in a variety with permutable congruences. In this setting, it is clear from 1.10 that every class is contained in a smallest radical class. We shall obtain a construction of the latter, based on the version of the Kurosh lower radical construction first used in [15].

Let $\Re$ be a non-empty class; without loss of generality we can take $\Re$ to be homomorphically closed. We define a class $\mathfrak{R}_{\alpha}$, for each ordinal number $\alpha$, as follows.

$\mathfrak{R}_{1}=\mathfrak{R} ; \quad \Re_{\alpha}=\{A \| A / \sigma \mid>1 \Rightarrow A / \sigma$ has a non-trivial congruence (that is, $\neq 0$ ) with all classes in $\left.\bigcup_{\beta<\alpha} \mathfrak{T}_{\beta}\right\}$, if $\alpha>1$. Note that $\mathfrak{R}_{\gamma} \subseteq \mathfrak{T}_{\alpha}$ if $\gamma \leqslant \alpha$.

5.1 TheOREM/DEFINITION. Let R be a non-empty homomorphically closed class in a congruence-permutable variety. Let

$$
L(\mathfrak{R})=\cup \mathbb{R}_{\alpha}, \text { where a extends over all ordinals. }
$$

Then

(i) $L(\mathscr{N})$ is a radical class and

(ii) $L(\mathfrak{N}) \subseteq \Re$ for every radical class $\Re$ with $\Re \subseteq \Re$.

$L(\mathscr{N})$ is called the lower radical class defined by $\mathfrak{K}$.

Proof. We first show that $L(\mathscr{N})$ is a radical class. By assumption, $\mathfrak{K}_{1}$ is homomorphically closed, and a simple transfinite induction argument establishes the homomorphic closure of the other $\mathfrak{N}_{\alpha}$. Thus $L(\mathfrak{N})$ is homomorphically closed. 
Let $A$ be an algebra with a congruence $\sigma$ such that $L(\mathscr{N})$ contains $A / \sigma$ and all $\sigma$-classes. Then there are ordinals $\delta$ and $\gamma_{a}, a \in A$ such that $A / \sigma \in \mathfrak{R}_{\delta}$ and $a \sigma \in \mathscr{R}_{\gamma_{a}}$ for each $a$. Let $\eta$ be an ordinal such that $\eta \geqslant \delta$ and $\eta \geqslant \gamma_{a}$ for each $a$. Then $A / \sigma$ and all the $a \sigma$ are in $\mathfrak{R}_{\eta}$. Let $\tau$ be any congruence on $A$ such that $|A / \tau|>1$. If $\sigma \leqslant \tau$, then, as $A / \sigma \in \mathfrak{R}_{\eta}$, we have $A / \tau \in \mathfrak{R}_{\eta}$. If $\sigma \$ \tau$, then as in the proof of 1.8 , for every $a \in A,(a \tau)[(\sigma \vee \tau) / \tau]$ is a homomorphic image of $a \sigma$, and one such class has cardinality greater than one. Thus $(\sigma \vee \tau) / \tau$ is non-trivial, and its classes are all in $\mathscr{R}_{\eta}$. We conclude that $A$ is in $\Re_{\eta+1}$, whence $L(\mathscr{R})$ satisfies (E).

Let $\left\{\sigma_{\lambda} \mid \lambda \in \Lambda\right\}$ be a chain of congruences on an algebra $A$ such that for each $\lambda$, every $\sigma_{\lambda}$-class is in $L(\mathscr{N})$. Let $\sigma=V_{\lambda \in \Lambda} \sigma_{\lambda}$, and let $T=a \sigma$ be any $\sigma$-class. Each $\sigma_{\lambda}$ induces a congruence $\sigma_{\lambda}^{\prime}$ on $T$ each class of which is a $\sigma_{\lambda}$-class and is thus in $L(\Re)$. If $\mu$ is a congruence on $T$ such that $|T / \mu|>1$, then for some $\lambda, \sigma_{\lambda}^{\prime} \leqslant \mu$. Then as in the previous argument, for each $t \in T,(t \mu)\left[\left(\sigma_{\lambda}^{\prime} \vee \mu\right) / \mu\right]$ is a homomorphic image of $t \sigma_{\lambda}^{\prime}$. Let $\xi_{\lambda}$ be an ordinal such that each $\sigma_{\lambda}^{\prime}$-class is in $\Re_{\xi_{\lambda}}$ (see the previous argument). Then each $\left(\sigma_{\lambda}^{\prime} \vee \mu\right) / \mu$-class is in $\Re_{\xi_{\lambda}}$. Let $\xi$ be an ordinal greater than all the $\xi_{\lambda}$. Then every non-trivial homomorphic image of $T$ has a non-trivial congruence with all its classes in $\mathfrak{K}_{\xi}$. Thus $T \in \mathfrak{R}_{\xi+1}$, so $a \sigma=T \in L(\Re)$. This proves that $L(\Re)$ satisfies $(\mathrm{C})$ and so is a radical class by 1.10 .

Now let $\Re$ be any radical class containing $\Re=\Re_{1}$, and let $\rho$ be the associated congruence. If $\mathfrak{T}_{\beta} \subseteq \Re$ for every $\beta<\alpha$, consider $A \in \mathfrak{R}_{\alpha}$. If $\left|A / \rho_{A}\right| \neq 1$, then $A / \rho_{A}$ has a non-trivial congruence with all its classes in $\cup_{\beta<\alpha} \Re_{\beta} \subseteq \Re$. This is impossible, so $\rho_{A}=1_{A}$ and $A \in \Re$. Thus $L(\Re) \subseteq \Re$ and the proof is complete.

\section{Examples}

6.B. Bands. Every band, that is, semigroup satisfying $x^{2}=x$, has a smallest congruence such that the corresponding factor band is a semilattice, that is, is commutative. Now it turns out that the congruence classes for the congruence referred to are rectangular [16], that is, satisfy the identity $x y x=x$. There are no non-trivial rectangular semilattices and it follows from this and a few other observations that the rectangular bands form a radical class whose corresponding semi-simple class is the class of semilattices. (Full details are given below.) This example provided the starting point for our investigation and a model for the definition of a radical class of idempotent algebras. By 3.2, if we accept that the 
semilattices form a semi-simple class, the variety defined by $x y=y x$ has attainable identities. In fact, Tamura [31] has provided the results which establish the following.

6.B.1. THEOREM [31]. The non-trivial varieties of bands which are semi-simple classes, that is, which have attainable identities are

(i) $\operatorname{VAR}(x y=y x)$ (semilattices),

(ii) $\operatorname{VAR}(x y=y)$ (right zero semigroups),

(iii) $\operatorname{VAR}(x y=x)$ (left zero semigroups).

All the varieties in 6.B.1 are defined by two-variable identities; we shall now classify all such varieties in terms of the concepts discussed in earlier sections. Each such variety is defined by a single identity; the following list of defining identities and names accounts for all the non-trivial ones. For a proof, see Petrich [24].

$$
\begin{aligned}
& x y=y x \quad(\text { semilattices }), \\
& x y=x y x \quad(\text { left regular semigroups }), \\
& y x=x y x \quad \text { (right regular semigroups), } \\
& x=x y x \quad \text { (rectangular bands), } \\
& x=x y \quad(\text { left zero semigroups }), \\
& y=x y \quad \text { (right zero seimgroups). }
\end{aligned}
$$

6.B.2. EXAMPLE. $\operatorname{VAR}(x=x y x)$ is a radical class and the corresponding semisimple class is $\operatorname{VAR}(x y=y x)$. To see this, we use 3.2 and 6.B.1 to see that $\operatorname{VAR}(x y=y x)$ is a semi-simple class, the corresponding radical class $\Re=$ $\left\{A \mid A / \sigma \in \operatorname{VAR}(x y=y x) \Rightarrow \sigma=1_{A}\right\}$ and the congruence $\rho$ associated with $\Re$ is given by

$$
\rho_{A}=\{\sigma \mid A / \sigma \in \operatorname{VAR}(x y=y x)\} .
$$

As noted above, $\rho_{A}$ has all its classes $\in \operatorname{VAR}(x=x y x)$. Thus for $A \in \Re$ (that is, for $\left.\rho_{A}=1_{A}\right) A$ itself is in $\operatorname{VAR}(x=x y x)$. On the other hand, it is clear that $\operatorname{VAR}(x=x y x) \subseteq \Re$.

6.B.3. EXAMPLE. $\operatorname{VAR}(x=x y)$ and $\operatorname{VAR}(y=x y)$ are radical classes. It is enough that we discuss the class $\operatorname{VAR}(x=x y)$ of left zero semigroups. If a band $A$ has a congruence $\sigma$ such that $A / \sigma$ and all $\sigma$ classes are in $\operatorname{VAR}(x=x y)$, then for $a, b \in A$, we have $a \sigma=(a \sigma)(b \sigma)=(a b) \sigma$, that is, $a \sigma a b$, so $a=a(a b)=a^{2} b$ $=a b$. Thus $\operatorname{VAR}(x=x y)$ satisfies $(\mathrm{E})$. Let $\tau, \mu$ be congruences on a band $B$ such 
that all $\tau$-classes and all $\mu$-classes are in $\operatorname{VAR}(x=x y)$. If $a, b \in B$ and $a(\tau \vee \mu) b$, then there exist $x_{1}, x_{2}, \ldots, x_{n} \in B$ such that

$$
a \tau x_{1} \mu x_{2} \tau x_{3} \mu \cdots \tau x_{n} \mu b \text {. }
$$

Since $a \tau x_{1}$, we have $a=a x_{1}$; since $x_{1} \mu x_{2}$, we have $x_{1}=x_{1} x_{2}$, and so on. Thus

$$
\begin{aligned}
a b & =a x_{1} b=a x_{1} x_{2} b=\cdots=a x_{1} x_{2} \cdots x_{n} b=\left(a x_{1} x_{2} \cdots x_{n-1}\right)\left(x_{n} b\right) \\
& =\left(a x_{1} x_{2} \cdots x_{n-1}\right) x_{n}=\cdots=a x_{1}=a .
\end{aligned}
$$

This shows that $\operatorname{VAR}(x=x y)$ satisfies $(J)$, so by $4.5, \operatorname{VAR}(x=x y)$ is a weak radical class. Let $\rho$ be the associated congruence. To show that $\operatorname{VAR}(x=x y)$ is actually a radical class, we obtain an explicit description of $\rho$.

Consider the relation $\lambda$ on a band $A$ define by the condition

$$
a \lambda b \Leftrightarrow a b=a \text { and } b a=b .
$$

As shown by McLean ([16], Lemmas 4 and 5), $\lambda$ is a right congruence. Let $\lambda^{*}$ be defined on $A$ by

$$
a \lambda^{*} b \Leftrightarrow a \lambda b \text { and } r a \lambda r b \quad \forall r \in A \text {. }
$$

Clearly $\lambda^{*}$ is an equivalence relation. If $a \lambda^{*} b$, then for any $s, r \in A$, we have

$$
r(s a)=(r s) a \lambda(r s) b=r(s b),
$$

so $s a \lambda * s b$. Furthermore $r a \lambda r b$ for all $r$, so, $\lambda$ being a right congruence,

$$
r(a s)=(r a) s \lambda(r b) s=r(b s),
$$

that is, $a s \lambda^{*} b s$, for all $s$. Thus $\lambda^{*}$ is a congruence. If $a \lambda^{*} b$, then, in particular, $a \lambda b$, so $a b=a$; hence all $\lambda^{*}$-classes are left zero semigroups. If $\sigma$ is any congruence on $A$, all of whose classes are left zero semigroups, then whenever $u \sigma v$ we have $u v=u, v u=v$, so $u \lambda v$. But then, since ruorv for every $r$, we have $r u \lambda r v$ for every $r$, that is, $u \lambda^{*} v$. Thus $\sigma \leqslant \lambda^{*}$. This proves that $\rho_{A}=\lambda^{*}$, that is,

$$
\forall A, a \rho_{A} b \Leftrightarrow a b=a, b a=b, r a r b=r a \text { and } r b r a=r b \text { for all } r \in A \text {. }
$$

From this characterization of $\rho$ it is clear that $f\left(\rho_{A}\right) \subseteq \rho_{B}$ for every surjective homomorphism $f: A \rightarrow B$. By $4.2, \operatorname{VAR}(x=x y)$ is a radical class.

6.B.4. EXAMPLE. Let $\Re_{l}$ be the class of bands $A$ satisfying the following condition:

$\forall a, b \in A \exists$ a finite sequence $x_{0}(=a), x_{1}, \ldots, x_{n}(=b)$ such that for each $i$, either $x_{i+1}$ is a right multiple of $x_{i}($ in $A)$ or $x_{i}$ is a right multiple of $x_{i+1}$.

Then $R_{1}$ is a radical class, and the corresponding semi-simple class is $\operatorname{VAR}(x=x y)$. (There is, of course, a corresponding result for $\operatorname{VAR}(y=x y)$.) As noted above, Tamura [31] has shown that $\operatorname{VAR}(x=x y)$ has attainable identities. By 3.2 and its 
proof, $\operatorname{VAR}(x=x y)$ is a semi-simple class and the corresponding radical class has as its associated congruence for each band $A$, the congruence $\wedge\{\sigma \mid A / \sigma \in$ $\operatorname{VAR}(x=x y)\}$. One now sees by reference to Lemma 2 of [31] (see also [7], [32]) that the radical class is $R_{l}$.

6.B.5. EXAMPLE. The class VAR $(x y=y x)$ of semilattices is a weak radical class but not a radical class. To see this, we first verify the conditions of 4.5 , beginning with $(E)$.

Let $\sigma$ be a congruence on a band $A$ such that $A / \sigma$ and all $\sigma$-classes are semilattices. If $a, b \in A$, then $(a b) \sigma(b a)$, so $(a b a) \sigma\left(b a^{2}\right)=b a$. Similarly $(b a b) \sigma a b$. Thus $(a b a) \sigma(b a b)$, so

$$
a b=(a b)^{3}=(a b a)(b a b)=(b a b)(a b a)=(b a)^{3}=b a,
$$

that is, $A$ is a semilattice. (This also follows from attainability, but the argument we have given, which uses only 2-associativity, may be useful elsewhere.)

Now let $\mu$ be a congruence on a band $A$ with each $\mu$-class a semilattice. If $a \mu b$, then for any $c \in A$ such that $b c=c b$, we have $(a c) \mu(b c)$ and $(c a) \mu(c b)$, so $(c a) \mu(a c)$. Since $(c a) \Re(c a c) \mathcal{L}(a c)$, we have $c a \mathscr{D} a c$, and hence cagac, $\Re, \mathfrak{L}, \mathscr{D}$ and $q$ being Green's relations on the semilattice $c a \mu$. Then $c a=a c$ (by [13], page 93).

If $\sigma$ and $\tau$ are congruences on a band $A$ and all $\sigma$-classes and all $\tau$-classes are semilattices, let $a, b \in A$ be such that $a(\sigma \vee \tau) b$. Then there are elements $x_{1}, x_{2}, \ldots, x_{n} \in A$ such that

$$
. a \sigma x_{1} \tau x_{2} \sigma x_{3} \tau \cdots \tau x_{n-1} \sigma x_{n} \tau b .
$$

Now $x_{1} x_{2}=x_{2} x_{1}$ and $a \sigma x_{1}$, so, as above, $a x_{2}=x_{2} a$. Then $x_{2} \sigma x_{3}$, so $a x_{3}=x_{3} a$, and so on. Thus $a b=b a$ and all ( $\sigma \vee \tau)$-classes are semilattices. This proves (J).

To see that $\operatorname{VAR}(x y=y x)$ is not a radical class, we consider the band $C$ with the following table:

\begin{tabular}{c|c|c|c|c} 
& $a$ & $b$ & $c$ & $d$ \\
\hline$a$ & $a$ & $b$ & $c$ & $d$ \\
\hline$b$ & $a$ & $b$ & $c$ & $d$ \\
\hline$c$ & $c$ & $d$ & $c$ & $d$ \\
\hline$d$ & $c$ & $d$ & $c$ & $d$
\end{tabular}

Let $\rho$ denote the congruence associated with $\operatorname{VAR}(x y=y x)$. Clearly the $\rho_{C}$-classes are $\{a, c\}$ and $\{b, d\}$. The set $I=\{c, d\}$ is an ideal of $C$. Let $\theta$ be the congruence on $C$ defined by the Rees factor band $C / I$. Then $\theta \vee \rho_{c} / \theta=1$. Since $C / I$ is not a semilattice, $\operatorname{VAR}(x y=y x)$ fails to satisfy $(\mathrm{R} 1)$, and so is not a radical class. 
6.B.6. ExAMPLE. $\operatorname{VAR}(x y=x y x)$ and $\operatorname{VAR}(y x=x y x)$ are weak radical classes but not radical classes. The demonstration follows the same pattern as that in the previous example; only the left regular case, that is, $\operatorname{VAR}(x y=x y x)$, will be considered.

Let $A$ be a band with a congruence $\sigma$ such that $A / \sigma$ and all the $\sigma$-classes are left regular. If $a, b \in A$, then $a b \sigma a b a$, so

$$
a b=(a b)^{3}=(a b)^{2}\left(a^{2} b\right)=(a b)(a b a)(a b)=(a b)(a b a)=(a b)^{2} a=a b a .
$$

Thus $A$ is left regular, so $\operatorname{VAR}(x y=x y x)$ satisfies (E).

Now let $\mu$ be a congruence on a band $A$ with all the $\mu$-classes left regular. If $b, c \in A$ are such that $b c=b c b$, then $c b=(c b)(c b)=c(b c b)=c b c$, while $(c b)(b c)(c b)=c b^{2} c^{2} b=c b c b=c b$ and $(c b)(b c)=c b^{2} c=c b c$. Thus we have

$$
b c=b c b \Rightarrow c b=c b c \text { and }(c b)(b c)=(c b)(b c)(c b) .
$$

Let $a \mu b$ and $b c=b c b$. Then $(c a) \mu(c b)$ and $(a c) \mu(b c)$, so making use of (*), we get

$$
\begin{aligned}
(c a) \mu & =(c a)^{2} \mu=\left(c a^{2} c^{2} a\right) \mu=[(c a)(a c)(c a)] \mu=[(c b)(b c)(c b)] \mu \\
& =[(c b)(b c)] \mu=[(c a)(a c)] \mu=(c a c) \mu .
\end{aligned}
$$

Thus $(c a) \mu(c a c)$, so that

$$
c a=(c a)^{3}=c a c a c^{2} a=c a(c a c) c a=c a(c a c)=(c a)^{2} c=c a c .
$$

Let $\sigma, \tau$ be congruences such that all $\sigma$-classes and all $\tau$-classes are left regular. If $a(\sigma \vee \tau) b$, then there are elements $x_{1}, x_{2}, \ldots, x_{n}$ of $A$ such that

$$
a \sigma x_{1} \tau x_{2} \sigma \cdots \tau x_{n-1} \sigma x_{n} \tau b .
$$

Now $a x_{1}=a x_{1} a$ and $x_{2} \tau x_{1}$, so as above $a x_{2}=a x_{2} a$. Since $x_{2} \sigma x_{3}$, we have $a x_{3}=a x_{3} a$, and so on. Thus after a few repetitions, we get $a b=a b a$. Every $\sigma \vee \tau$-class is therefore left regular, so $\operatorname{VAR}(x y=x y x)$ satisfies $(\mathrm{J})$. Hence $\operatorname{VAR}(x y=x y x)$ is a weak radical class.

Since semilattices are left regular, the congruence $\rho_{C}$ on the band $C$ of $6 . B .5$ has left regular classes. Since $C / \rho_{C}$ is a right zero semigroup, $\rho_{C}$ is the largest congruence on $C$ with left regular classes. Now $C / I$ is not left regular, and as in 6.B.5, it follows that $\operatorname{VAR}(x y=x y x)$ is not a radical class.

$A$ variety with attainable identities satisfies (E) in the contexts we are dealing with and is closed under extensions - the exact analogue of $(E)$-in varieties of rings and groups with operators. Some counterexamples in ring-based structures, given in [10] and [11], show that the converse is false for this kind of universal class. Our examples discussed so far provide similar counterexamples. 
6.B.7. EXAMPLE. In the variety of all bands, the subvarieties of rectangular bands, left regular bands and right regular bands satisfy (E) but do not have attainable identities.

In varieties of ring and group-based structures [9] and in congruence-permutable varieties of idempotent algebras (3.5) subvarieties with attainable identities are SSR classes. The case of semilattices shows that the converse is false for idempotent algebras in general.

6.B.8. EXAMPLE. In the variety of bands, the subvariety of semilattices has attainable identities, but is not an SSR class.

In view of Tamura's theorem (6.B.1) above and 3.4 we can now state the following.

6.B.9. THEOREM. The proper SSR-classes of bands are $\operatorname{VAR}(x=x y)$ and $\operatorname{VAR}(y$ $=x y)$.

We can summarize the information obtained about two-variable definable varieties of bands by the following table.

\begin{tabular}{|c|c|c|c|c|c|}
\hline $\begin{array}{c}\text { Defining } \\
\text { identity }\end{array}$ & (E) & $\begin{array}{c}\text { Weak radical } \\
\text { class }\end{array}$ & $\begin{array}{c}\text { Radical } \\
\text { class }\end{array}$ & $\begin{array}{c}\text { Attainable } \\
\text { identities }\end{array}$ & SSR class \\
\hline$x y=y x$ & Yes & Yes & No & Yes & No \\
\hline$x y=x y x$ & Yes & Yes & No & No & No \\
\hline$y x=x y x$ & Yes & Yes & & No & No \\
\hline$x=x y x$ & Yes & Yes & Yes & No & No \\
\hline$x=x y$ & Yes & Yes & Yes & Yes & Yes \\
\hline$y=x y$ & Yes & Yes & Yes & Yes & Yes \\
\hline
\end{tabular}

6.D. Idempotent distributive groupoids. We next consider (multiplicatively written) groupoids for which the multiplication is idempotent and left and right distributive over itself, that is, which satisfy the identities

$$
x^{2}=x ; \quad x(y z)=(x y)(x z) ; \quad(x y) z=(x z)(y z) .
$$

We shall present two examples in the radical theory of idempotent distributive groupoids, which we shall call ID groupoids.

In the case of bands, the variety of semilattices has attainable identities and the corresponding radical class has as its associated congruence the two-sided Green's equivalence $\mathscr{f}$ ([13], page 93$)$. 
6.D.1. EXAMPLE. In the variety of ID groupoids, the subvariety

$$
\operatorname{VAR}((x y) z=x(y z), x y=y x)
$$

of semilattices has attainable identities and is therefore a semi-simple class. Let $\Re$ denote the corresponding radical class, $\rho$ the associated congruence. Then for $a, b \in A$, we have

$$
a \rho_{A} b \Leftrightarrow a \text { and } b \text { generate the same ideal of } A \text {. }
$$

Most of what we need to establish this is stated without proof by Ruedin [25]; we include the details for the sake of completeness. Let $\mathscr{V}=\operatorname{VAR}((x y) z=$ $x(y z) ; x y=y x)$, and let $g$ be the two-sided Green's relation, defined as for semigroups. Let $A$ be an ID groupoid.

If $a, b \in A$, then $a b=a b^{2}=(a b)(a b)=(a(a b))(b(a b))=(a(a b))\left((b a) b^{2}\right)$, from which it follows that $a b g b a$. If $a, b, c \in A$, then $(a b) c=(a c)(b c)=$ $(a(b c))(c(b c))$, whence $(a b) c \mathscr{G} a(b c)$. Thus $A / \mathcal{F} \in \mathcal{V}$.

Let $B$ be a $f$-class of $A, a \in B$. Then any $b \in B$ generates the same ideal of $A$ as $a$; in particular $b$ is in the ideal generated by $a$. Hence $b$ can be obtained from $a$ by a finite number of left and right multiplications by elements of $A$, that is, $b$ will look something like this:

$$
\cdots\left(x_{3}\left(\left(a x_{1}\right) x_{2}\right)\right) x_{4} \cdots
$$

If $A$ is mapped onto a semilattice $\bar{A}$, then $B$ is taken onto a subsemilattice $\bar{B}$ in which, denoting the images of $a, b$ by $\bar{a}, \bar{b}, \bar{b}$ is a multiple of $\bar{a}$ (and similarly $\bar{a}$ is a multiple of $\bar{b}$ ). But then $|\bar{B}|=1$. It follows that $\mathcal{g}=\kappa^{\Upsilon}$ in the sense of Section 2 . The argument just given also shows that no $G$-class can be mapped onto a semilattice at all. Thus $\operatorname{VAR}((x y) z=x(y z), x y=y z)$ has attainable identities and the corresponding radical class has $f$ as its associated congruence.

The class $\mathfrak{R}$ of 6.D.1 may be regarded as analogous to the class of rectangular bands. In our second example, we treat a counterpart of the left zero semigroups. We first introduce some notation. For an element $a$ of an ID groupoid $A$, we denote left multiplication by $L_{a}$.

\section{D.2. EXAMPLE. Let}

$$
\Re=\left\{A \mid a, b \in A=\Rightarrow L_{a}^{n}(b)=\text { a for some } n\right\} .
$$

Then $\Re$ is a radical class. Let $A$ be an ID groupoid. We consider the relation $\sigma$ on $A$ defined by

$$
a \sigma b \Leftrightarrow L_{a}^{n}(b)=a \quad \text { for some } n \quad \text { and } \quad L_{b}^{m}(a)=b \quad \text { for some } m \text {. }
$$


(Of course, without loss of generality we can take $m=n$.) Clearly $\sigma$ is reflexive and symmetric. Let $a=L_{a}^{n}(b), b=L_{b}^{k}(c)$. Then

$$
\begin{aligned}
a & =L_{a}^{n}\left(L_{b}^{k}(c)\right)=L_{a}^{n-1} L_{a}\left(L_{b}^{k}(c)\right)=L_{a}^{n-1} L_{a b}^{k}(a c)=L_{a}^{n-2} L_{a} L_{a b}^{k}(a c) \\
& =L_{a}^{n-2} L_{a(a b)}^{k}(a(a c))=L_{a(\cdots(a(a b)) \cdots)}^{k}(a(\cdots(a(a c)) \cdots)),
\end{aligned}
$$

where there are $n a$-factors in each product, that is

$$
a=L_{L_{a}^{n}(b)}^{k}\left(L_{a}^{n}(c)\right)=L_{a}^{k}\left(L_{a}^{n}(c)\right)=L_{a}^{k+n}(c) .
$$

It follows that $\sigma$ is transitive.

If $a=L_{a}^{n}(b)$, then for any $d \in A$,

$$
\begin{aligned}
d a & =d(a(\cdots(a(a b)) \cdots))=(d a)(\cdots(d a)((d a)(d b)) \cdots) \\
& =L_{d a}^{n}(d b)
\end{aligned}
$$

and

$$
\begin{aligned}
a d & =(a(\cdots(a(a b)) \cdots)) d=(a d)(\cdots(a d)((a d)(b d)) \cdots) \\
& =L_{a d}^{n}(b d),
\end{aligned}
$$

from which it follows that $\sigma$ is a congruence. Clearly all $\sigma$-classes are in $R$, and $\sigma \geqslant \tau$ for every congruence $\tau$ with all its classes in $\Re$, that is, (R2) holds. If $\bar{a} \sigma \bar{b}$, where $\bar{a}, \bar{b} \in A / \sigma$, then for some $n, \bar{a}=L_{\bar{a}}^{n}(\bar{b})$, that is, $a \sigma L_{a}^{n}(b)$, where $a \in \bar{a}$, $b \in \bar{b}$. But then for some $n$, we have

$$
a=L_{a}^{m}\left(L_{a}^{n}(b)\right)=L_{a}^{m+n}(b),
$$

so $a \sigma b$, that is, $\bar{a}=\bar{b}$. Hence (R3) holds also. Since $\Re$ is homomorphically closed, it is weak radical class. The proof that $\Re$ is actually a radical class is now like that in 6.B.3.

6.P. Congruence-permutable varieties. Finally we consider some examples in varieties where congruences permute. Radical classes are much easier to find, or at any rate to recongnize, in such situations: see the characterization in 1.10 . We have already considered one way of constructing radical classes - the lower radical construction in Section 5 . We now consider upper radicals defined by classes $\Re$ satisyfing $\left(\mathrm{Sl}^{\prime}\right)$ of Section 2.

6.P.1. THEOREM/DEFINITION. Let $\Re$ be a class satisfying $\left(\mathrm{Sl}^{\prime}\right)$ in a congruencepermutable variety. Let $\Re=\left\{A \mid A / \sigma \in \mathfrak{R} \Rightarrow \sigma=1_{A}\right\}$. Then $\Re$ is a radical class. $\Re$ is called the upper radical class defined by $\mathfrak{R}$.

Proof. Clearly $\Re$ is homomorphically closed. Let $A$ have a congruence $\sigma$ such that $\Re$ contains $A / \sigma$ and all the $\sigma$-classes. Suppose $A / \tau \in \mathfrak{R}$. If $\sigma \neq \tau$, then $(\sigma \vee \tau) / \tau$ is non-trivial, so some $(\sigma \vee \tau) / \tau$-class has a non-trivial homomorphic 
image in $\mathscr{T}$. But as in the proof of 1.8 , each $(\sigma \vee \tau) / \tau$-class is a homomorphic image of a $\sigma$-class, so we have a contradiction. Thus $\sigma \leqslant \tau$. But then the map $A \rightarrow A / \tau$ factors through $A / \sigma$, so $|A / \tau|=1$. Thus $A$ is in $\Re$ and $\Re$ satisfies (E).

Finally, let $\sigma=V_{\alpha \in \Lambda} \sigma_{\alpha}$, where the $\sigma_{\alpha}$ form a chain of congruences on an algebra $A$ and all $\sigma_{\alpha}$-classes are in $\Re$ for each $\alpha$. Let $T$ be a $\sigma$-class. Then each $\sigma_{\alpha}$ induces a congruence $\sigma_{\alpha}^{\prime}$ on $T, \vee \sigma_{\alpha}^{\prime}=1_{T}$. If $T / \tau \in \mathfrak{R}$, then as in the previous part of the proof, every $\left(\sigma_{\alpha}^{\prime} \vee \tau\right) / \tau$-class, as a homomorphic image of a $\sigma_{\alpha}^{\prime}$-class, is in $\Re$ and hence $\sigma_{\alpha}^{\prime} \leqslant \tau$. But then $1_{T}=\vee \sigma_{\alpha}^{\prime} \leqslant \tau$; it follows that $T \in \Re$, so $\Re$ satisfies $(C)$. By $1.10, \Re$ is a radical class.

6.P.2. CoRollary. If $\mathscr{T}$ is hereditary, then $\Re($ as defined in 6.P.1) is a radical class.

6.P.3. COROLLARY. If on is a class of simple algebras then $\Re$ (as defined in 6.P.1) is a radical class.

We consider just one more general source of radical classes in a congruencepermutable variety.

6.P.4. THEOREM. Let $*$ be a binary derived operation, let $p(x, y)=x^{*} y$, $p^{2}(x, y)=p(p(x, y), y), \ldots, p^{n+1}(x, y)=p\left(p^{n}(x, y), y\right)$, and let

$$
\Re=\left\{A \mid a, b \in A \Rightarrow \exists n \text { such that } b=p^{n}(a, b)\right\} .
$$

Then $\Re$ is a radical class.

Proof. Again we verify that $R$ satisfies the conditions of 1.10 . Clearly $R$ is homomorphically closed. If $A$ has a congruence $\sigma$ such that $A / \sigma$ and all $\sigma$-classes are in $\Re$, then for $a, b \in A$ there is an $n$ such that $b \sigma=p^{n}(a \sigma, b \sigma)=p^{n}(a, b) \sigma$. Then for some $m$ we have $b=p^{m}\left(p^{n}(a, b), b\right)=p^{m+n}(a, b)$, so $A$ is in $\Re$ and $R$ satisfies (E). Since $R$ is closed under directed unions, we get $(C)$ as for varieties in 3.1.

An affine module over a ring $R$ with identity is a structure defined on a unital right $R$-module by the operations

$$
\omega=\omega_{a_{1}, a_{2}, \ldots, a_{n}} ; \quad a_{i} \in R, \quad \Sigma a_{i}=1 ; \quad \omega\left(u_{1}, u_{2}, \ldots, u_{n}\right)=\Sigma u_{i} a_{i} .
$$

See, for example, [5] or [29] for details; for an internal characterization see Ostermann and Schmidt [23]. The variety Aff- $R$ of affine $R$-modules has permutable congruences, as can be seen either from the tenary term

$$
\psi(x, y, z)=x-y+z
$$


[3] or the fact that the affine module congruences are precisely the module congruences on the "same" module. Thus 1.10 characterizes the radical classes in Aff- $R$. They were also investigated, from a quite different point of view, and characterized by Szendrei [29]. It is, in fact, quite easy to find a correspondence between radical classes in Aff- $R$ and those in the variety Mod- $R$ of unital right $R$-modules.

Varieties of quasigroups, algebras with three binary operations,., \and / such that

$$
y \backslash(y x)=(x y) / y=y(y \backslash x)=(x / y) y=x
$$

have permutable congruences; see, for example, [3], page 79.

We consider one example of a radical class in the variety of idempotent groupoids, those satisfying the additional identity $x^{2}=x$ (and hence also the identities $x \backslash x=x$ and $x / x=x)$.

\section{P.5. EXAMPLE. For idempotent quasigroups, let}

$$
x^{*} y=y \backslash[(y x) x]
$$

and let $p^{n}(x, y)$ be defined as in 6.P.4. Then

$$
\Re=\left\{A \mid a, b \in A \Rightarrow b=p^{n}(a, b) \text { for some } n\right\}
$$

is a nontrivial radical class.

If we take a subvariety $\mathcal{V}$ of idempotent quasigroups, then $\mathscr{V} \cap \mathcal{R}$ is a radical class in $\mathcal{T}$. As an example, consider the variety $\mathcal{V}_{M}$ of medial idempotent quasigroups: these satisfy the extra identity

$$
(x y)(z w)=(x z)(y w) .
$$

Now it has been shown by Csákány and Megyesi [6] that $\mathcal{V}_{M}$ is equivalent to Aff- $P$, where $P$ is the ring of rational expressions of the form $f(x) / x^{k}(1-x)^{l}$ over the integers. Because of the closure properties characterizing radical classes, this equivalence (described in [6], pages 18-19) takes radical classes to radical classes. The radical class $\mathcal{V}_{M} \cap \mathbb{R}$ corresponds to the radical class

$$
\left\{A \mid a, b \in A \Rightarrow \exists n \text { such that }(1+x)^{n} a+\left(1-(1+x)^{n}\right) b=b\right\},
$$

which in turn corresponds to the class of $(1+x)$-primary modules in Mod-P. An investigation of radical theory in one of $\mathcal{V}_{M}$, Aff- $P$ and Mod- $P$ could possibly throw some light on radical theory in the others; this seems worthy of further consideration. (There appears to be a misprint on page 18 of [6]: the equations $v(1-x)=1, v^{\prime}=1-v$ should, it seems, be $v^{\prime}(1-x)=1, v=1-v^{\prime}$.) 
Note that the radical class of 6.D.2 is of the type described in 6.P.4. However the proof that this class is a radical class is much more complicated, as congruences need not permute in idempotent distributive groupoids. (For a counterexample, see [26], page 143, Exemple.)

\section{Acknowledgements}

Some of the results presented here were obtained while the author was visiting the United States as part of a University of Tasmania Outside Studies Programme, and with the partial support of a Fulbright Senior Scholar Award. Special thanks must go to Tom Hall and Ed Scheiblich for their invaluable assistence with the crucial Example 6.B.5.

The referee detected a couple of errors and made a number of suggestions for improvements. The careful examination which gave rise to those discoveries and suggestions is greatly appreciated by a neophyte in the unfamiliar world of general algebras.

\section{References}

[1] A. V. Arhangel'skii and R. Wiegandt, 'Connectednesses and disconnectednesses in topology', General Topology and Appl. 5 (1975), 9-33.

[2] G. Birkhoff, Lattice theory (Amer. Math. Soc. Colloq. Publ. XXV, 1948).

[3] S. Burris and H. P. Sankappanavar, A course in universal algebra (Springer, 1981).

[4] A. H. Clifford, 'Radicals in semigroups', Semigroup Forum 1 (1970), 103-127.

[5] B. Csákány, 'Varieties of affine modules', Acta Sci. Math. (Szeged) 37 (1975), 3-10.

[6] B. Csákány and L. Megyesi, 'Varieties of idempotent medial quasigroups', Acta Sci. Math. (Szeged) 37 (1975), 17-23.

[7] P. Dubreil, 'Contribution à la théorie des demi-groupes (II),' Rend. Mat. (5) 10 (1951), 183- 199.

[8] E. Fried and R. Wiegandt, 'Connectednesses and disconnectednesses in graphs,' Algebra Universalis 5 (1975), 411-428.

[9] B. J. Gardner, 'Semi-simple radical classes of algebras and attainability of identities,' Pacific J. Math. 61 (1975), $401-416$.

[10] B. J. Gardner, 'Extension-closed varieties of rings need not have attainable identities,' Bull. Malaysian Math. Soc. (2) 2 (1979), 37-39.

[11] B. J. Gardner, 'Extension-closure and attainability for varieties of algebras with involution,' Comment. Math. Univ. Carolinae 21 (1980), 285-292.

[12] H.-J. Hoehnke, 'Radikale in allgemeinen Algebren,' Math. Nachr. 32 (1966), 347-383.

[13] J. M. Howie, An introduction to semigroup theory (Academic Press, 1976).

[14] S. R. Kogalovskii, 'Structural characteristics of universal classes,' Sibirsk. Mat. Ž. 4 (1963), 97-119 (in Russian).

[15] A. G. Kurơs, 'Radicals in the theory of groups,' Colloq. Math. Soc. János Bolyai 6 (Rings, Modules and Radicals, Keszthely, 1971), 271-296.

[16] D. McLean, 'Idempotent semigroups,' Amer. Math. Monthly 61 (1954), 110-113.

[17] A. I. Mal'cev, The metamathematics of algebraic systems (North-Holland, 1971). 
[18] L. Márki, 'Radical semisimple classes and varieties of semigroups with zero,' Colloq. Math. Soc. János Bolyai 20 (Algebraic Theory of Semigroups, Szeged, 1976), 357-369.

[19] L. Márki, R. Mlitz and R. Strecker, 'Strict radicals of monoids,' Semigroup Forum 21 (1980), 27-66.

[20] R. Mlitz, 'Kurosch-Amitsur-Radikale in der universalen Algebra,' Publ. Math. Derecen 24 (1977), 333-341.

[21] R. Mlitz, 'Radicals and semi-simple classes of $\Omega$-groups,' Proc. Edinburgh Math. Soc. (2) 23 (1980), 37-41.

[22] H. Neumann, Varieties of groups (Springer, 1967).

[23] F. Ostermann and J. Schmidt, 'Der baryzentrische Kalkül als axiomatische Grundlage der affinen Geometrie,' J. Reine Angew. Math. 224 (1966), 44-57.

[24] M. Petrich, 'A construction and a classification of bands,' Math. Nachr. 48 (1971), 263-274.

[25] J. Ruedin, 'Equivalences de Green et demi-treillis images homomorphes maximales d'un groupoide distributif,' C. R. Acad. Sci. Paris Sér. A 264 (1967), 429-432.

[26] J. P. Soublin, 'Étude algébrique de la notion de moyenne,' J. Math. Pures Appl. 50 (1971), 53-264.

[27] R. Strecker, ' $M$-Radikale von universalen Algebren,' Publ. Math. Debrecen 26 (1979), 245-254.

[28] F. Szász, 'On radicals of semigroups with zero I,' Proc. Japan Acad. 46 (1970), 595-598.

[29] A. Szendrei, 'Torsion theories in affine categories,' Acta Math. Acad. Sci. Hungar. 30 (1977), $351-369$.

[30] T. Tamura, 'Attainability of systems of identities on semigroups,' J. Algebra 3 (1966), 261-276.

[31] T. Tamura, 'Note on attainability of identities on bands,' J. Algebra 28 (1974), 1-9.

[32] G. Thierrin, 'Sur le théorie des demi-groupes,' Comment. Math. Helv. 30 (1956), 211-223.

[33] R. Wiegandt, Radical and semisimple classes of rings (Queen's University, Kingston, Ontario, 1974).

\author{
Mathematics Department \\ University of Tasmania \\ G.P.O. Box $252 \mathrm{C}$ \\ Hobart, Tasmania 7001 \\ Australia
}

